\title{
Las relaciones entre los actores en las políticas de desarrollo de las aglomeraciones urbanas francesas $(1980-2000)$
}

Christian Lefèvre *

A pesar del proceso de descentralización emprendido, hace ahora casi dos decenios, Francia sigue representando en el exterior del hexágono el modelo de un Estado centralizador, dirigista y jacobino. Esta imagen sigue siendo cierta desde muchos puntos de vista, incluso cuando están en curso evoluciones significativas hacia un mejor reparto de las responsabilidades entre los actores de las políticas públicas. Antes de entrar en una presentación y en un análisis de la evolución de las políticas de desarrollo de las aglomeraciones urbanas francesas a través de las relaciones entre actores, conviene fijar los elementos del contexto en que estas políticas han evolucionado desde principios de los años ochenta (sección 1). Abordaremos a continuación las políticas de desarrollo urbano en tres secciones. La primera tratará de la evolución de la naturaleza de los problemas que se presentan hoy en día a la ciudad y las primeras medidas para hacerles frente. En una segunda sección, nos centraremos en las innovaciones locales y sus dificultades de inserción en los marcos de elaboración y puesta en marcha de las políticas públicas todavía deficientes. Una tercera sección se interesará por las recientes evoluciones jurídicas y políticas iniciadas en 1999 y 2000 y por su impacto en la manera de actuar las políticas de desarrollo urbano en los próximos años.

\section{El contexto}

Francia es todavía un Estado unitario en el sentido de que, a corto plazo, al menos, las leyes de la República continúan aplicándose al conjunto del territorio nacional y de que todavía no hay previsto un sistema derogatorio o particular para determinadas partes del territorio ${ }^{1}$. La organización institucional francesa se basa en el sistema napoleónico en el que a cada nivel de entidad local (Región, Departamento, Municipio) corresponde una estructura desconcentrada del Estado, representada a nivel de las regiones y de los departamentos por las psefecturas, a nivel infradepartamental por las subprefecturas y a nivel de los municipios por los ayuntamientos que son, al mismo tiempo, elegidos locales y representantes del Estado.

El proceso de descentralización se inició a mediados de los años setenta con una globalización progresiva de las ayudas centrales hacia las entidades locales. Se concretó principalmente en las Leyes de descentralización de 1982 y de 1983, que transfirieron un importante número de competencias del Estado y de sus estructuras desconcentradas hacia los ejecutivos locales, principalmente a los consejos generales en los departamentos 


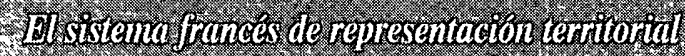
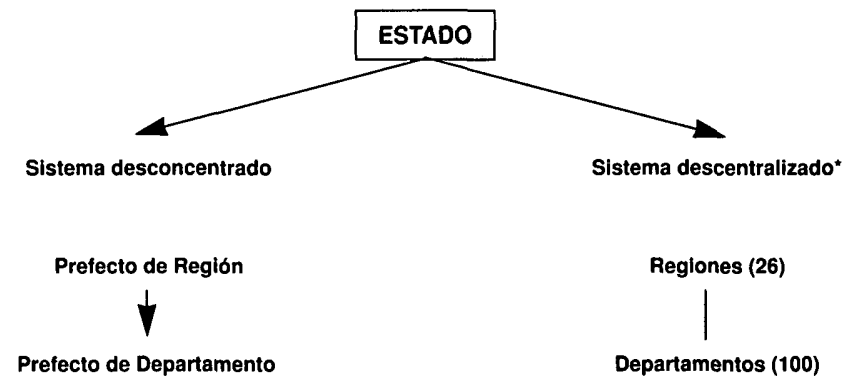

Alcalde

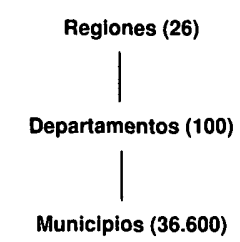

* No existe jerarquía entre las entidades territoriales.

y a los ayuntamientos en los municipios. La única innovación institucional de las leyes de descentralización fue el establecimiento de las regiones en tanto que entidades territoriales, lo que llegó a ser realidad en 1986 con la elección por sufragio universal directo de los ejecutivos regionales.

El Estado francés mantiene un papel central innegable en el seno de la sociedad francesa, que se explica por la historia de la formación del Estado-nación a partir, por lo menos, del Antiguo Régimen. En efecto, el Estado se construyó contra los señores feudales, luego, progresivamente, contra las entidades locales y principalmente contra las ciudades. Desconfiado de los «poderes» locales, se ha opuesto, durante siglos, a todo intento de emancipación de las entidades territoriales que, por el contrario, ha mantenido cuidadosamente limitadas, controladas y dirigidas. Es dentro de este marco como debe entenderse hoy la evolución de la descentralización francesa que es un proceso todavía joven, incompleto y, en parte, discutido.

El tema urbano está en la agenda política desde principios de los años sesenta y ha sido y es, todavía, en gran parte, conducido por el Estado. El territorio nacional está compuesto por más de 36.000 municipios, lo que fragmenta considerablemente las áreas urbanas. Consciente desde el principio de los años sesenta del desfase entre los territorios municipales y los territorios funcionales de las áreas urbanas, el Estado ha tratado de favorecer las cooperaciones entre los municipios con el fin de desarrollar unas políticas públicas establecidas sobre unos territorios considerados idóneos. La elección de la cooperación intermunicipal (que se denomina intermunicipalidad en sus formas político-institucionales) es fundamental. En efecto, contrariamente a lo que ha ocurrido en otros países, principalmente en el Reino Unido en los años setenta o en Italia desde 1990, el Estado francés se ha negado siempre a crear nuevos entes territoriales a nivel de las aglomeraciones urbanas con el fin de evitar que se pudieran establecer contrapoderes a su autoridad. $\mathrm{Ha}$ sido ayudado en ello por los municipios

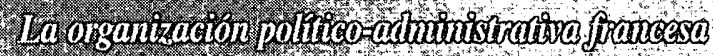

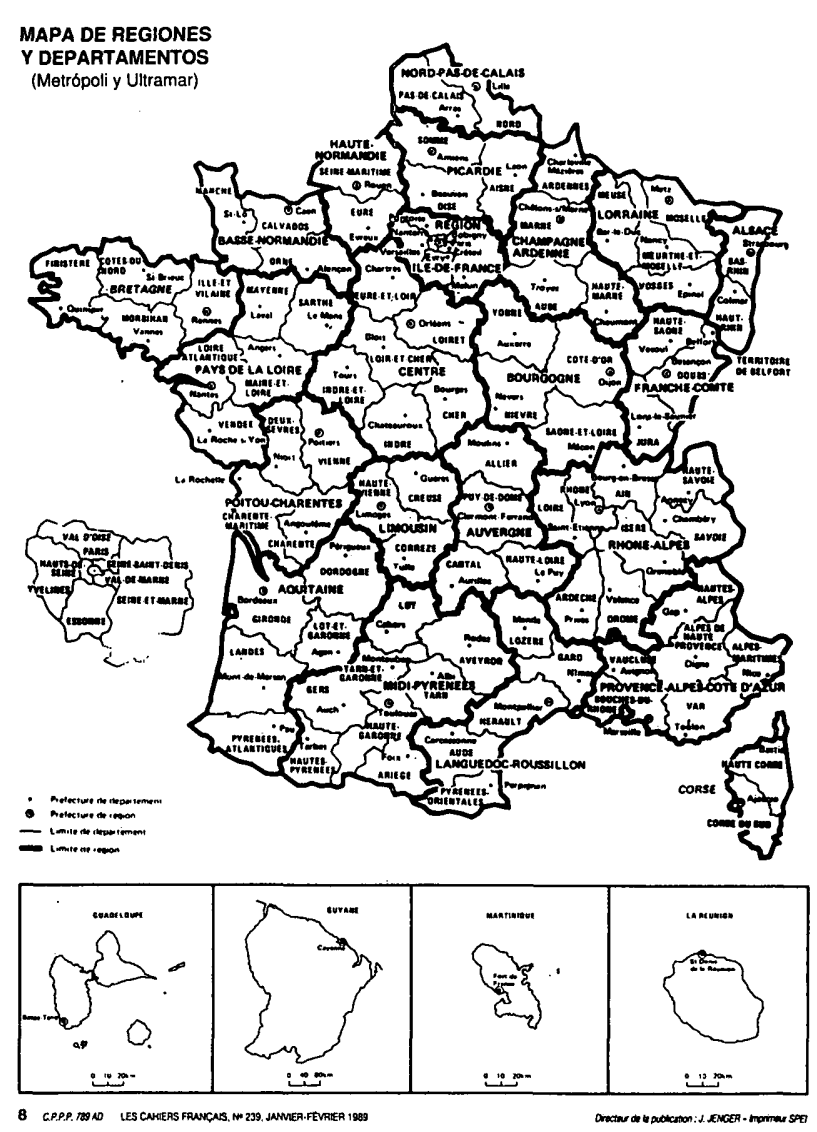

y los departamentos, celosos del poco poder de que disponían. A partir de entonces se han multiplicado las estructuras municipales, que cuentan hoy con cerca de 20.000 instituciones.

Las relaciones entre los actores públicos y los actores privados, sean éstos las empresas o las estructuras de representación de los habitantes, han sido siempre desequilibradas en la medida en que el sector público (y en primer lugar el Estado) se ha situado como guía, incluso como único actor legítimo para asegurar la dirección de la sociedad y consecuentemente la dirección de las políticas públicas. Por eso el Estado ha creado sus propios interlocutores y socios en la sociedad civil, ya sea en el mundo de la empresa estableciendo las cámaras consulares (cámaras de comercio y de industria, cámaras de oficios y del artesanado) o en el mundo social, definiendo así un corporativismo a la francesa. En el sector de los servicios urbanos, Francia ha iniciado desde principios de siglo Xx un sistema de relaciones originales, la gestión delegada, que otorga a la entidad pública la organización general de los servicios así como el control de su ejecución, pero que atribuye el funcionamiento real a las empresas privadas. En consecuencia, el sector privado ocupa hoy en Francia un lugar importante en la gestión de las 
ciudades, lo que distingue a Francia de los países llamados de socialismo municipal como los Países Bajos, Italia, Suiza o Alemania.

De esta breve presentación resulta un elemento fundamental. En Francia, las políticas a nivel local son históricamente, en primer lugar, las políticas del Estado. La descentralización ha permitido a las entidades locales, especialmente las más poderosas en cuanto más beneficiadas por las leyes de descentralización, como las grandes ciudades y los departamentos, intervenir de manera legítima en las políticas locales y sobre todo en las políticas urbanas. Para ello, los actores públicos han creado unas estructuras adecuadas. Es el caso del Estado que constituyó después de la guerra la Caisse des Dépôts et Consignations (CDC), establecimiento público que administra los ahorros de los franceses, pero sobre todo que interviene, gracias a este colosal recurso financiero, en las políticas de desarrollo urbano (construcciones inmobiliarias, creación de equipamientos y de infraestructuras, principalmente). Las CDC se han asociado a las entidades locales para crear sociedades de economía mixta (SEM), cuyo capital es totalmente público y que participan activamente en las políticas urbanas. En segundo lugar, el Estado interviene por mediación de organismos ad hoc, como los establecimientos públicos de ordenación, concebidos para desarrollar núcleos enteros de aglomeraciones urbanas francesas y ello en cooperación más o menos importante con las entidades territoriales. Además, el Estado está muy presente en las ciudades por mediación de sociedades nacionales como Electricité et Gaz de France o como la Société Nationale des Chemins de Fer Français (SNCF), empresas importantes no sólo por sus actividades, sino también por su patrimonio.

Tradicionalmente, el dominio del sector público en las politicas urbanas está establecido por ley. En 1967, período en que el Estado manda en las políticas urbanas, se aprueba la Loi d'Orientation Foncière (LOF), que servirá de marco para la acción pública urbana hasta el presente. Esta ley fija para los próximos decenios los marcos jurídicos y los instrumentos de la ocupación del suelo. Crea también los Schémas Directeurs d'Aménagement et d'Urbanisme (SDAU), documentos en principio iniciados y aprobados por el Estado, pero hoy controlados, completamente, por las entidades locales a partir de la descentralización. En principio, se trataba de documentos de localización de equipamientos e infraestructuras que fijan a medio plazo el desarrollo de las aglomeraciones urbanas en la medida en que sus territorios de intervención tratan de adaptarse a los territorios funcionales. A estos SDAU deben conformarse los Plans d'Occupation des Sols (POS), documentos municipales encargados de poner en marcha, a nivel municipal, las orientaciones contenidas en los SDAU. La LOF estableció, así, una jerarquía en los documentos de urbanismo dando la supremacía a los SDAU, pero sin conferirles un estatuto de oponibilidad jurídica. En efecto, los planes directores no se pueden oponer a terceros, cualidad que, sin embargo, se reconoce a los POS. Con el paso del tiempo la relación entre estos dos documentos con estatuto jurídico diferente va a plantear bastantes problemas.

La LOF estableció igualmente una nueva estructura encargada de la planificación a nivel de las aglomeraciones urbanas: las agencias de urbanismo. Se trata de estructuras de estudio y de asesoramiento a las entidades locales, en un principio controladas por el Estado, pero que van a pasar progresivamente a manos de las entidades locales. Hoy día existen agencias de urbanismo en la mayor parte de las aglomeraciones urbanas francesas. Su desarrollo ha jugado un papel muy importante en la experiencia de las entidades locales en las politicas urbanas.

A finales de los años ochenta, el panorama de los actores de las políticas urbanas está bastante bien delimitado. El sector privado está muy presente pero en un registro esencialmente operativo. La elaboración, la dirección y la puesta en marcha de las políticas de desarrollo urbano son realmente un asunto de interés del sector público y, principalmente, del Estado, aunque la descentralización hace evolucionar las responsabilidades efectivas de unos y de otros. La década que acaba de terminar puede marcar un giro en muchos aspectos, pero no es posible todavía confirmar cuáles. En efecto, aunque el Estado trata de reconfigurar su papel, nada hay que haga pensar que esta reconfiguración se efectuará en una línea de retirada progresiva, como se puede constatar en Italia, por ejemplo. Algunos elementos podrían prefigurar hoy, a la inversa, una «vuelta del Estado». Ciertamente, las entidades locales demandan más responsabilidades, pero ciserán capaces de procurarse los medios? Aunque las autoridades públicas en su conjunto parecen ponerse de acuerdo para entreabrir la puerta a los sectores privado y asociativo, el sistema de actores de las políticas continúa otorgando un papel motor y central a los poderes públicos. Éste es un primer análisis de las transformaciones en curso al que luego volveremos a fin de apoyar estos primeros análisis.

\section{Las ciudades francesas. Nuevos problemas y respuestas confusas
de los poderes públicos}

A principio de los años ochenta, las ciudades francesas, como la mayor parte de las ciudades del mundo, se hallaban enfrentadas a nuevos problemas en un momento en que los problemas viejos no habían encontrado soluciones satisfactorias en un contexto económico poco favorable. 
Los nuevos problemas se sitúan en campos también nuevos, donde la acción pública no disponía de ninguna experiencia sólida. La crisis económica puso en evidencia la crisis más profunda de un sistema de acción basado en el papel central del Estado en un período en que las entidades locales pasan por las primeras experiencias de una descentralización completamente nueva. Las primeras algaradas estallan en las periferias de las grandes ciudades (Lyon, Marseille, Paris). La violencia urbana destapa los errores de un urbanismo fundado en la masificación como la política de las Grands Ensembles y las Zones à Urbaniser en Priorité (ZUP) del período de crecimiento demográfico, urbano y económico de los Gloriosos Treinta. Sin que se pueda hablar de fractura social, expresión que será utilizada con provecho por el candidato J. Chirac para ganar las elecciones presidenciales de 1995, la integración social de la sociedad francesa se debilita. Pero los problemas aparecen, igualmente, en otros sectores como el medio ambiente (contaminación del aire y del agua, deterioro de los espacios naturales en el centro de las ciudades) o la salud.

Estos problemas vienen a unirse a otros que no han encontrado soluciones. El tema de los transportes, en el centro de los movimientos sociales urbanos de los años setenta, resurgió con un desarrollo automovilístico familiar sin precedentes mostrando la incapacidad del sistema de infraestructuras para atender un número cada vez más grande de usuarios y de mercancías, debido principalmente al escalonamiento urbano que contribuye a aumentar considerablemente las distancias domicilio-trabajo (entre 1975 y 1990, la distancia del trayecto medio del domicilio al trabajo se ha duplicado, pasando de 7 a 14 $\mathrm{kms}$ ). En el centro de las ciudades, la crisis económica puso de relieve el declive industrial. El desempleo que se genera está asociado a la crisis industrial que se traduce en la multiplicación de los baldíos. El desarrollo económico se identifica con la reconversión de los baldíos industriales, verdaderos vacíos urbanos que conviene revitalizar.

Estos problemas, viejos y nuevos, ponen a prueba la capacidad de los actores públicos, y en primer lugar del Estado, para responder, es decir, para diagnosticarlos y resolverlos. En efecto, estos problemas cuya naturaleza es multi o transectorial tropiezan con las estructuras técnico-administrativas del aparato del Estado y de otras autoridades públicas organizadas sobre bases sectoriales. Para hacer frente a los problemas urbanos, es preciso hacer cooperar a los diferentes sectores de la acción pública; resolver el problema de los transportes y de los desplazamientos, por ejemplo, exige la cooperación entre las direcciones de la red de comunicaciones, de los transportes y del urbanismo, estructuras muy independientes las unas de las otras dentro de la administración municipal. Lo mismo sucede a nivel del Estado donde los Ministerios de Transportes, de Equipamiento, de Medio Ambiente y de Interior están obligados a cooperar. La transectorialidad de los problemas pone en tela de juicio, pues, la organización interna de las autoridades públicas.

Pero estos mismos problemas ponen de relieve también las insuficiencias de la organización institucional urbana, en la medida en que el diagnóstico y las soluciones sólo pueden actuar válidamente en territorios que sobrepasan en gran medida los de las municipalidades. Ello, a pesar del considerable número de estructuras intermunicipales, que hasta principios de los años noventa siguen siendo en su mayor parte sectoriales (sindicatos con vocación única o SIVU) y abarcan la mayoría de las veces unos territorios limitados en relación con los problemas a afrontar. La organización y el mapa intermunicipal francés presentan un desfase respecto a los problemas a tratar.

Por último -y se trata probablemente del elemento más delicado, puesto que afecta al fundamento mismo de la legitimidad de las entidades públicas-, el desarrollo de los problemas antes mencionados hace que poco a poco las entidades tomen conciencia de su incapacidad para afrontarlos solas. Desde entonces, el sistema de actores, cuidadosamente limitado al sector público, al menos en su fase de toma de decisiones, debe abrirse a los que poseen una experiencia, un savoir faire y una legitimidad específica en estos campos. Es, pues, la concepción misma del papel y del lugar de los actores públicos en las políticas públicas lo que se pone en tela de juicio.

Consciente de la gravedad de los problemas, limitados, en un primer momento, al fenómeno de las banlieues, el Estado optó por dar una doble respuesta: una respuesta institucional por medio de la creación de nuevas estructuras de dirección en el seno del gobierno y del aparato administrativo nacional y una respuesta en términos de políticas públicas mediante el establecimiento de lo que hoy se conoce como la politique de la ville.

De forma continuada, aunque vacilante, el Estado crea en 1988 la Direction Interministérielle à la Ville (DIV), que a partir de 1990 estará situada bajo la responsabilidad del primer Ministerio para la ciudad. En 1992, crea, a nivel local, una administración desconcentrada, encargada muy especialmente de la política de la ciudad: el «subprefecto para la ciudad». Con el establecimiento de estas tres innovaciones institucionales, el Estado trata de crear un nuevo enfoque multisectorial, integrado, de su acción. El Ministerio para la ciudad es, en efecto, no sectorial. Esto está simbolizado por su primer ministro, M. Delabarre, ministro de Estado, ciertamente, pero voluntariamente sin presupuesto real, puesto que la idea es que las medidas que se tomen sean financiadas con los recursos provenientes de los diferentes Ministerios (Asuntos Sociales, Interior, Equipamiento y Transporte). La creación de la DIV sigue la misma lógica. En los primeros años, la DIV no dispone de ningún presupuesto propio, pero se surte de los presupuestos de los diferentes Ministerios. 
La política de la ciudad va a suponer para el Estado la ocasión de una innovación de tipo organizativo en el medio urbano. Consciente de su incapacidad para asumir por sí solo las políticas necesarias para responder a los problemas de las áreas periféricas, debido, principalmente, a la descentralización y a la crisis de las finanzas públicas, el Estado aplica a la ciudad una nueva forma de llevar a cabo las políticas públicas a través de la "contractualización», inaugurada en el marco de los primeros Contrats de Plan État-Regions (CPER) entre 1984 y 1988. Los CPER establecieron una sociedad entre las entidades territoriales y el Estado para los temas de planificación y de ordenación del territorio. La gestión es descentralizada y contractual y está dirigida para las colectividades locales por las regiones. En el marco de la política de la ciudad, las autoridades públicas (básicamente el Estado a través del Ministerio para la Ciudad y los municipios interesados) se ponen de acuerdo sobre los objetivos y las medidas a poner en marcha en el marco de un documento específico firmado por los socios. Nacen así los primeros «contratos de la ciudad» a principios de los años noventa cuya forma de gestión va a ser adoptada, luego, en otros sectores y que llega hasta nuestros días.

Más que una innovación, esta forma de gestión. se lleva a cabo con un sistema de actores limitado al sector público. Aunque los medios asociativos son consultados, no participan directamente en la elaboración de las políticas y de las acciones. Sólo intervienen en la fase previa, en tanto que facilitadores de recursos, de información y de capacidad de diagnóstico. Lo mismo sucede en el sector del desarrollo económico en el que la política de la ciudad invierte marginalmente y donde las empresas sólo están escasamente implicadas. Aunque hay innovación, se limita a la esfera pública con la creación, por ejemplo, a nivel local, de Missions de Développement Urbain, especie de células de reflexión y de puesta en marcha de la política de la ciudad, compuestas por profesionales de orígenes diversos (sociólogos, arquitectos, economistas, urbanistas) que trabajan, conjuntamente, en sectores hasta ahora completamente separados. Igualmente sucede con los contratos de la ciudad, en los que los comités de dirección siguen dominados por los actores públicos, esencialmente el Estado desconcentrado y las entidades locales, principalmente los municipios.

Estas primeras respuestas a los problemas urbanos son muy desalentadoras (Commissariat Général du Plan, 1993b). Efectivamente, son muy desordenadas, Estado y entidades locales avanzan caóticamente en sus gestiones, alli donde se hace necesario «aprender actuando». El contenido y el marco de las politicas que se deben desarrollar son con mucho una labor de aficionados. Se pasa, así, del Développement Social des Quartiers (DSQ) al Développement Social Urbain (DSU), faltando, para ese cambio de denominación, un paso del barrio a la ciudad (es decir, en realidad, al municipio) y una ampliación de los sectores de la política de inversiones (empleo, lucha contra la delincuencia, rehabilitación de viviendas, construcción de infraestructuras y de equipamientos). El desorden es también el síntoma de una primera crisis de juventud de la descentralización (Commissariat Général du Plan, 1993a). En efecto, las Leyes de descentralización de 1982 y 1983 establecieron bloques de competencias propios para cada entidad local en un sistema político-institucional donde no hay jerarquía entre los distintos niveles de gobierno. Para el municipio, el urbanismo operativo; para el departamento, el desarrollo económico y la ayuda social; para la región, la formación profesional. La realidad es completamente diferente. Aparece, al contrario, cada entidad local interviniendo en campos «reservados» a otros, sin concertación ni verdadera cooperación, en un sistema que A. Mabilau ha llamado el de los «socios-rivales» (MaBLLEAU, 1991).

Esta evolución de las políticas y de las relaciones entre los actores se realiza en un momento en que los nuevos protagonistas aparecen en la escena urbana. Para ciertos autores (LORRAIN, 1995), la descentralización ha dejado el campo libre a los grandes grupos de servicios urbanos, principalmente a la Lyonnaise des Eaux o a la Compagnie Générale des Eaux, empresas líderes mundiales en este sector. En efecto, muchos ayuntamientos aprovechan las competencias transferidas por las leyes de descentralización para otorgar a estos grandes grupos un lugar más importante en los servicios urbanos. Hoy día estas empresas, en un sistema de gestión delegada, se ocupan de los transportes públicos, del tratamiento de basuras domésticas, del suministro de agua, del saneamiento, de la gestión de los aparcamientos, de la teleasistencia, pero también de la pro. moción inmobiliaria, de la telemática, incluso en algunos casos de la construcción y de la gestión de infraestructuras y de equipamientos a través de sus filiales. Han levantado grandes imperios y se imponen a los elegidos locales como unos socios obligados de las políticas urbanas.

En el año 1992 la descentralización cumple la primera década. Ésta muestra también sus límites, su falta de conclusión, y hace comprender especialmente al Estado que todavía le queda por jugar un papel en las políticas públicas y más ampliamente en la dirección de las sociedades locales. En efecto, durante diez años, los servicios del Estado han vivido mal la descentralización. Despojadas, en gran parte, de sus poderes, pero sobre todo de su legitimidad de acción a nivel local, las administraciones desconcentradas (en primer lugar las Directions Départementales de l'Equipement (DDE) del poderoso Ministère de l'Equipement et des Travaux Públics) han permanecido en un plano discreto, inseguras del lugar que debían ocupar en las políticas locales. El principio de los años noventa marca un despertar del Estado que intenta recuperar una posición central y una postura nueva utilizando un instrumento tradicional de los gobiernos: la ley. En efecto, a través de la promulgación de dos leyes, la Ley sobre la Administración Terri- 
torial de la República (Loi ATR) de 1992 y la Ley de Orientación para la Ordenación y el Desarrollo del Territorio (LOADT) de 1995, el Estado volvió a investir el campo del desarrollo territorial a través de lo que se denominan políticas constitutivas, es decir, las políticas que fijan los marcos y los procedimientos para que se relacionen los actores sin imponer el contenido, descentralización obliga.

La Ley ATR se quiere que sea una nueva ley de descentralización. Repleta de innovaciones (por ejemplo, creación de «ententes» interregionales, legalización de referéndums locales, creación de comités de usuarios de los servicios), nos interesa aquí, directamente, por las transformaciones que impone a los cuadros políticos e institucionales de desarrollo urbano. Los diez años que acaban de finalizar han influido en las mentalidades y un número determinado de altos funcionarios y de elegidos locales se dan cuenta de que es conveniente «imprimir una mayor velocidad» si se quiere que la política de la ciudad y las políticas de desarrollo económico y de empleo den sus frutos. Lo que pasa por una mayor integración de las políticas sectoriales y por la elaboración y la puesta en marcha de estas politicas en los territorios más idóneos, dejando en evidencia a algunos municipios.

Se van a utilizar dos instrumentos para alcanzar estos objetivos: uno institucional y otro fiscal. En el aspecto institucional, la Ley ATR crea dos nuevas estructuras intermunicipales, la comunidad de municipios y la comunidad de ciudades, que sólo pueden ser establecidas sobre una base voluntaria. Estas dos formas intermunicipales son nuevas y suponen una verdadera revolución en el sistema institucional local. En efecto, la intermunicipalidad francesa ha sido, hasta aquí, una intermunicipalidad de gestión porque se interesaba, en primer lugar, por la gestión común de los servicios cuyas características técnicas explicaban la necesidad de una cooperación entre municipios (saneamiento, agua, transportes públicos, tratamiento de residuos, etc.). El establecimiento de las comunidades de municipios y de las comunidades de ciudades transforma la intermunicipalidad francesa en una intercomunalidad de proyecto, porque las competencias asignadas a estos dos tipos de estructuras exigen que las municipalidades cooperen a fin de definir conjuntamente proyectos que comprometan en el futuro de sus territorios. De esta manera, la Ley ATR otorga unas competencias obligatorias a las comunidades de municipios y de ciudades: desarrollo económico, medio ambiente y ordenación; a estas competencias obligatorias se deben añadir un cierto número de competencias opcionales entre las que encontramos: cultura, transporte público o vivienda. Las comunidades de municipios están reservadas a las zonas rurales o a las aglomeraciones urbanas de menos de 20.000 habitantes, mientras que las comunidades de ciudades se dirigen a las ciudades más grandes.

En el aspecto fiscal, la innovación es grande. La Ley ATR crea un impuesto profesional único (TPU) en el territorio de las comunidades, TPU que se entrega a la estructura intermunicipal, proporcionándole así los medios y una relativa autonomía para establecer sus políticas. Se trata de un avance importante en la medida en que en los municipios urbanos, al menos, el impuesto profesional representa el recurso fiscal más importante y es donde este impuesto profesional siempre ha sido objeto de una competencia desenfrenada de los municipios con el fin de atraer el mayor número de empresas a su territorio. La TPU es obligatoria para las comunidades de ciudades; su fijación, en cambio, se ha dejado a la libre elección de las comunidades de municipios. En este marco, las comunidades de municipios aparecen como una forma menos apremiante que las comunidades de ciudades para las municipalidades afectadas.

La Ley ATR es, pues, fundamental para la creación de nuevos marcos político-insitucionales de las políticas de desarrollo local. Sin embargo, desde muchos puntos de vista resulta tímida, debido a que institucionaliza una asociación público-público y no integra al sector privado en una gestión y un sector, el desarrollo económico y la ordenación, donde ocupa, sin embargo, una lugar importante.

En 1995, la Ley de Orientación y Desarrollo del Territorio (LOADT) pretende, a la vez, terminar con el laissez-faire o el no intervencionismo del Estado durante la primera década de la descentralización y volver a las grandes políticas nacionales de ordenación del territorio de los años sesenta y setenta. En 1993, el nuevo ministro del Interior, C. Pasqua, exige la incorporación del sector de ordenación territorial a su ministerio. Su máxima ambición es promulgar una ley que establezca los fundamentos de una nueva política de ordenación a nivel nacional. Por eso la ley votada en 1995 será una ley de orientación, en la medida en que fija las políticas nacionales sobre la materia para los años venideros.

C. Pasqua rompe con el discurso y las acciones titubeantes de los gobiernos precedentes, dado que tiene una visión real del problema de la ordenación del territorio en Francia y del papel que debe jugar el Estado. Heredero de la tradición «gaullista» de un Estado fuerte, conserva, también, una concepción bastante rural de la sociedad francesa. Su ley de ordenación del territorio es una ilustración de ello. Para él, el tema de la ordenación del territorio se basa en el desequilibrio entre París (y la región de Île-de-France) y el resto de Francia. El gran problema es la desertización de los campos franceses. Para hacerle frente, la LOADT decide establecer un plan nacional de ordenación y de desarrollo del territorio en el que la cuestión de la accesibilidad es central, puesto que el Estado afirma que todas las partes del territorio no podrán encontrarse en el año 2015 a más de $50 \mathrm{kms}$ o a más de 45 minutos en automóvil de una entrada de autopista o de una estación TGV (art. 17 de la LOADT). Además, la ley crea un nuevo territorio de acción pública, el pays, que representa un espacio que posee 
una cohesión geográfica, cultural, económica o social en el que las entidades territoriales van a definir un proyecto común de desarrollo. El pays puede llegar a ser con el tiempo el nuevo territorio de cooperación entre las entidades locales a partir del momento en que estas últimas se pongan de acuerdo para emprender un proyecto colectivo. La DATAR estima que los pays van a ocupar una posición institucional intermedia entre los municipios y los departamentos. La noción de pays se dirige, en primer lugar, al mundo rural.

El Estado se proyecta también en esta recomposición ins. titucional y territorial. En primer lugar, afirma que va a reorganizar sus servicios desconcentrados teniendo en cuenta la creación de los pays por las colectividades locales. En segundo lugar, establece un procedimiento nuevo, la Directiva Territorial de Ordenación (DTA), que se refiere a los territorios que el Estado considera como un reto nacional. Se trata de vastas extensiones de terreno que pueden abarcar varios departamentos sobre las cuales el Estado fija las orientaciones en materia de ordenación, de localización de las grandes infraestructuras de transporte y de grandes equipamientos. Sobre estos territorios, la elaboración de una DTA se efectúa bajo la responsabilidad del Estado junto con las regiones, los departamentos y los municipios (así como sus agrupaciones). Las DTAs se imponen a los planes directores. Hay, pues, claramente por la mediación de las DTAs una voluntad por parte del Estado de inmiscuirse en la cooperación entre las entidades locales y de asumir un papel central sobre los grandes retos territoriales.

La LOADT ha sido muy mal acogida por las ciudades, principalmente por las más grandes, que la consideran como un grave error por parte del Estado que no ha sabido darse cuenta del cambio de la sociedad francesa y, especialmente, del hecho de que se trata de una sociedad completamente urbana. En un informe titulado «Mon pays c'est ma ville», la Asociación de Alcaldes de las Grandes Ciudades de Francia (AMGVF, 1995) denuncia violentamente el ruralismo de la LOADT que olvida pura y simplemente a la gran mayoría de la población francesa. Para la AMGVF, aunque algunas DTAs se refieran a determinadas zonas urbanas (el Grand Lyon, la metrópoli Nice-Toulon, Nantes-Saint Nazaire), el problema urbano está ausente del debate sobre la ordenación del territorio cuando, por el contrario, se trata del problema más importante que Francia y el Estado deben afrontar en este fin de siglo. Defendiendo la opinión contraria a la de la LOADT, la AMGVF propone reformas profundas de los marcos de la acción pública territorial y medidas importantes en pro de políticas urbanas de gran amplitud: multiplicación de los créditos, reforma de la fiscalidad local en beneficio de los agrupamientos de municipios, elección por sufragio universal directo de los ejecutivos de las comunidades urbanas y de las comunidades de ciudades o de municipios, etc., medidas que van a ser, progresivamente, puestas en la agenda política del gobierno socialista a partir de 1997.

\section{La innovación local a prueba de las carencias de los cuadros y de los instrumentos de las políticas públicas urbanas}

Si bien en los años noventa, los marcos de la acción colectiva evolucionan aunque continúan situándose en un paradigma en el que lo público es dominante, algunas ciudades comienzan, sin embargo, a experimentar una transformación significativa en las relaciones entre actores económicos y actores políticos. Ciertamente, en muchos casos, las relaciones entre estas dos esferas son débiles. Aunque el sector privado y, especialmente, las grandes empresas no son invitadas más que en contadas ocasiones a la mesa de discusión de los proyectos públicos importantes, esto no significa, sin embargo, que ambas esferas no mantengan relaciones entre ellas, sino que estas relaciones van por otros canales, más discretos, más informales, pero igualmente eficaces. Con todo, llega un momento en que uno de los socios, a veces los dos, desean dar a sus relaciones una forma más institucionalizada, más visible. Las cámaras de comercio y de industria (CCI) han constituido durante mucho tiempo la estructura mediante la cual el sector privado participaba en los debates públicos y podía, así, aportar su punto de vista en los proyectos urbanos y comunicar sus propios mensajes. De esta forma, la década de los 90 ha visto nacer algunas iniciativas sin marco legal en algunas aglomeraciones urbanas.

La metrópoli de Lille (más de 1 millón de habitantes) constituye una buena ilustración de los acercamientos entre el sector privado y las autoridades locales en las políticas urbanas. En 1993, el Presidente de la CCI, un gran industrial con influencia, creó el Comité Grand Lille. Se trata de una asociación informal de los principales empresarios de la aglomeración urbana, cuyo objetivo es sopesar directamente las opciones estratégicas de desarrollo del territorio de Lille en un período en que los retos son numerosos (llegada del TGV, apertura del Euro Tunel, candidatura de los Juegos Olímpicos de 2004, etc.). Considerando que los elegidos locales tienen dificultad para definir y, sobre todo, para poner en ejecución una política coherente que permita aprovechar estas oportunidades, estos empresarios deciden aportar formalmente sus ideas y sus críticas a las políticas que se discuten. El Comité Grand Lille se compone de varios centenares de miembros y organiza forums y debates con los actores sociales y políticos de la aglomeración. Llega a ser, así, un socio poderoso y fijo de los poderes públicos hasta el punto de que la comunidad urbana de Lille decidió en abril de 1999 trans- 
formarlo en consejo de desarrollo, especie de asamblea consultiva parecida a nivel de la metrópoli al conjunto de fuerzas económicas y sociales, prefiguración de lo que unos meses más tarde llegará a ser una verdadera institución establecida por la nueva ley sobre la ordenación del territorio (véase más abajo).

En Nantes (500.000 habitantes), la gestión ha sido diferente, puesto que se ha hecho a iniciativa de los elegidos locales. En efecto, en 1999, el alcalde de Nantes, Presidente del Distrito, decidió constituir una Conférence Consultative de l'Agglomération Nantaise (CCAN). Esta estructura aparece también como un conjunto de las fuerzas vivas económicas y sociales y obtiene enseguida el apoyo de la cámara de comercio local y de los comités de expansión. Se unen también los sindicatos. Los círculos económicos no son correo de relaciones con los elegidos locales, pero acompañan la gestión en un período en que la metrópoli de Nantes reflexionaba sobre su futuro con el lanzamiento por los electos del proyecto Nantes 2005 en que la cuestión del desarrollo económico es central.

Los ejemplos de Lille y de Nantes no son los únicos en Francia, pero representan los casos más acabados de un acercamiento entre los actores económicos y políticos en la elaboración y la puesta en práctica de políticas de desarrollo territorial, aun cuando no citemos nada más que a las estructuras consultivas, los canales de influencia más clásicos, que probablemente sean, todavía, los más utilizados. Por eso, estas evoluciones indican una transformación profunda a la vez en las relaciones entre medios políticos y económicos locales, pero también en el interior del sistema político espacial de las aglomeraciones urbanas. Por un lado, si bien la dirección de las políticas locales permanece en manos de los elegidos, estos últimos admiten que los medios empresariales deben también tomar parte directa y legítimamente en los debates que comprometen el futuro de los espacios locales. En lo que se refiere a los empresarios, los cambios son también profundos. Por una parte, aceptan, al menos algunos, entrar en diálogos regulares, transparentes y significativos con el mundo político; por otra, adıniten que las estructuras tradicionales de representación del mundo económico, cerca de con los poderes públicos, como las cámaras consulares (CCI, Chambres de métiers, etc.), no son suficientes para agotar esta representación que se encuentra así directamente discutida desde el interior. Del lado de los políticos, el paso dado pasa por una cooperación intermunicipal necesaria que necesita ser intensificada. Es, asimismo, significativo que sean los alcaldes del centro de las ciudades quienes hagan gestiones de colaboración con sus periferias, demostrando así que las políticas de desarrollo hoy día no pueden hacerse cadla una «en su propio terreno». El sistema de actuación de las metrópolis francesas se ha abierto, pues: abierto a la cooperación entre los elegidos locales y abierto al mundo económico y social. Las dos principales leyes de 1999 y la Ley SRU de 2000 (véase a continuación) no hacen sino sacar partido $y$ enseñanzas de estas transformaciones.
A pesar de todo, los cambios operados en el transcurso de los últimos veinte años en los marcos y los instrumentos de las políticas de desarrollo resultan insuficientes para responder a los retos urbanos. Numerosas carencias perduran a nivel de la organización institucional, del rol y de la doctrina del Estado, del sistema fiscal, de los instrumentos de gestión del urbanismo y de puesta en relación de los medios económicos y políticos.

En el plano institucional, persisten dos problemas. En primer lugar, la confusión que reina en las relaciones entre las entidades locales; luego, un relativo fracaso de intermunicipalidad. Hemos visto que el sistema de los bloques de competencias no ha podido evitar una confusión de los papeles respectivos de cada nivel territorial. De hecho, todos se ocupan un poco de todo. Más allá del sistema de bloques de competencias, esto se explica por la ausencia de jerarquía entre los gobiernos locales y después de liderazgo. Se asiste más bien a una maraña de competencias que no es en sí perjudicial, puesto que esta situación corresponde a una preocupación de las entidades territoriales de tratar los problemas en sus dimensiones intersectoriales. Además, este barullo competencial se explica por la necesidad de incrementar la colaboración entre las autoridades públicas por razones finan. cieras, no siendo capaz ninguna de ellas por sí sola de sacar adelante una política de desarrollo. A esto se añade el hecho de que las regiones tratan de invertir en nuevos campos de la acción pública a fin de colocarse como socios ineludibles, reforzando así su peso político en la arquitectura institucional. $\mathrm{El}$ problema proviene del hecho de que ninguna ley de descentralización o de mejora de la descentralización ha venido a ofrecer un marco estable de relaciones entre los gobiernos locales. Las propuestas del Commissariat Général du Plan con ocasión de la preparación del $\mathrm{X}^{\mathrm{o}}$ plan que pretendía establecer en un territorio dado un dirigente, un líder, lo que, quizá, hubiera permitido una mejor dirección de las políticas de desarrollo, no se han llevado a cabo.

Por el lado de las relaciones horizontales, principalmente entre los municipios, las numerosas leyes sobre la materia no han podido, sin embargo, resolver los problemas de pertinencia a la vez de medios y de territorios para la operatividad de las políticas. Los nuevos organismos públicos de cooperación intermunicipal (EPCI) creados por la Ley ATR han tenido un éxito moderado. Por un lado, se han constituido muy pocas comunidades de ciudades; sólo se han creado cuatro y, únicamente, en aglomeraciones urbanas de tamaño pequeño. La comunidad más grande de ciudades, la de la Rochelle, sólo cuenta con 100.000 habitantes. Por otro lado, aunque las comunidades de municipios se han multiplicado (entre 1993 y 1999 se han creado más de 1.300), éstas se encuentran, principalmente, en las zonas rurales. En tercer lugar, las principales metrópolis francesas han preferido optar por las comunidades de municipios más que por las comunidades de ciudades, manifestando, así, un enfriamiento en su deseo de cooperación, ya que las comu- 
nidades de municipios son menos apremiantes (el establecimiento de un régimen tributario propio es voluntario, mientras que es obligatorio para las comunidades de ciudades) para las municipalidades que son sus miembros. Se han creado situaciones «absurdas», como en Marsella, segunda aglomeración urbana francesa donde el territorio funcional está compartido por tres EPCI: una comunidad de municipios, Marsella-Metrópoli, que sólo agrupa a tres municipios, Marsella, situada entre una comunidad de ciudades al este, comandada por el municipio de Aubagne, y al norte por otra comunidad de municipios en torno a la ciudad-central de Aix en Provence: iTres EPCI que disponen de competencias idénticas y que se hacen la competencia en un territorio funcional muy independiente, pues disponen sólo de un único canal de empleos y de desplazamientos!

El Estado no ha sabido o no ha querido intervenir de manera coherente: Las políticas estatales que se han multiplicado en el marco de la contractualización con los diferentes niveles de entidades se han traducido en una proliferación de circunscripciones territoriales, que han acabado en una verdadera división de la acción pública. Zonas francas urbanas de la Ley de Orientación para la Ciudad, Grandes proyectos Urbanos, Zonas de Educación Prioritarias (ZEP) del Ministerio de Educación Nacional, territorios de contratos de la ciudad, programas locales del hábitat (PLH), por no citar más que éstos, a los cuales han venido a unirse las zonas de las políticas europeas, han acrecentado la fragmentación de los territorios urbanos. Las DTAs creadas para que el Estado aporte una relativa coherencia en las políticas públicas sólo abarcan determinadas aglomeraciones urbanas y no siempre han sido elaboradas. Más que nunca la acción pública está fragmentada territorialmente y en su contenido y el Estado no se decide, todavía, a constituir verdaderas estructuras capaces de considerar globalmente a las zonas urbanas. Ahora bien, como vemos, la actuación libre y voluntaria de las entidades locales no conduce a más coherencia. En un país unitario e históricamente centralizado como Francia, la coherencia no parece poder venir nada más que del Estado, que se revela incapaz de desempeñar ese papel.

En el plano fiscal, la situación es también difícil. Hace más de veinte años que se habla de una reforma de la fiscalidad local, fiscalidad fundada en cuatro impuestos (los «cuatro viejos») creados algunos de ellos hace más de un siglo. El período de crecimiento y el de la competitividad territorial actual, agravado por el proceso de descentralización, han hecho de los recursos fiscales un reto importante para los municipios. Especialmente, el impuesto profesional, que es el principal ingreso fiscal del municipio, ha sido objeto de una competencia importante entre los municipios de una misma aglomeración urbana. En efecto, este impuesto que grava a las empresas lleva a cada municipio a atraer a su territorio el mayor número de ellas jugando con el tipo de los impuestos; cuanto más bajo sea el tipo de los impuestos, más empresas estarán interesadas en instalarse en el municipio en cuestión; cuanto más numerosas sean las empresas; más podrán los municipios permitirse bajar los tipos de sus impuestos profesionales, disponiendo, así, de ingresos suficientes para hacerlo, lo que contribuiría a hacer todavía más atractivo su territorio para las empresas; y así sucesivamente. En este contexto, las municipalidades se han lanzado a una competencia, en ocasiones feroz, creando de manera desordenada, durante los años ochenta y principios de los noventa, zonas de actividades susceptibles de atraer empresas. El resultado ha sido un despilfarro de dinero público, una multiplicación de las zonas de actividades industriales o del artesanado en una misma aglomeración urbana, haciéndose la competencia entre ellas para atraer a las mismas empresas. Durante los años noventa, se han multiplicado los informes de los poderes públicos y de las cámaras de comercio para denunciar este efecto perverso del sistema fiscal y de là descentralización, subrayando la falta de cooperación entre las municipalidades y la brecha abierta en el seno de la ciudad entre los municipios ricos que pueden atraer a las empresas más eficaces y las menos contaminantes, y los municipios pobres condenados a perder empleos y actividades.

El Estado no se ha quedado quieto en ese debate, pero no ha osado comprometerse en una acción importante orientada a suprimir los efectos perversos de la fiscalidad local. Es cierto que la Ley ATR ha permitido que se desarrolle una mutualización del impuesto profesional (el impuesto profesional único de la aglomeración urbana), pero esta mutualización resultó marginal hasta mediados de los años noventa, dejada a la libre elección de las entidades locales en un marco institucional, como hemos visto, confuso y complejo.

La dirección del desarrollo urbano se ha visto también, en gran medida, contrarrestada por una progresiva obsolescencia de los documentos de planificación iniciados por la LOF. Muy pronto, los Planes de Ocupación del Suelo (POS) quedaron desfasados con unos Esquemas Directores basados en un crecimiento demográfico y económico que no se produjo con la amplitud prevista. Aparecieron otros problemas como el debilitamiento industrial y sus efectos espaciales como la multiplicación de baldíos en el centro de las aglomeraciones urbanas. El desfase entre los POS y los Esquemas Directores es más grave por cuanto los dos documentos principales del urbanismo estaban pensados para articularse de manera armoniosa. El gran período de revisión de los Esquemas Directores iniciado a finales de los años ochenta y que termina ahora, ha producido nuevos documentos de planificación sin continuidad real con los viejos esquemas. Actualmente, aunque muchas aglomeraciones urbanas francesas disponen de Esquemas Directores; se trata de hecho de documentos de marketing territorial cuyo objetivo no es la localización de equipamiento e infraestructuras, sino la puesta en imagen de las prioridades de desarrollo de 
la aglomeración urbana sin contenido preciso y sin deseo real de localización de esas prioridades. En este marco, la articulación entre POS y SDAU no tiene mayor interés. Estos dos documentos resultan, pues, obsoletos, tanto en su concepción como en su cobertura espacial. En efecto, la mayor parte de los nuevos Esquemas Directores no se adaptan a los territorios funcionales de las ciudades, sino que resultan con mucha frecuencia tributarios de los territorios de los antiguos SDAU.

En paralelo a estas evoluciones, han aparecido otros documentos de planificación con sus especificidades y territorialidades propias: Planes de desplazamientos urbanos, Programas Locales del Hábitat, planes de protección de zonas verdes, etc., se han venido a añadir a los documentos existentes sin un auténtico deseo de buscar una coherencia. En resumen, la planificación territorial pensada para traer armonía al desarrollo urbano ha dejado poco a poco el puesto a una yuxtaposición de planes sin articulación entre ellos.

A todas estas deficiencias de los marcos de la acción pública y probablemente debido, en parte, a ellas, viene a unirse una carencia de otro tipo que se puede definir como una carencia de asociaciones estratégicas, principalmente entre actores económicos y actores políticos. Ya hemos planteado esta cuestión, pero quisiéramos insistir aquí sobre la debilidad del Estado en este campo. Mientras que durante los años sesenta y setenta, el Estado promovió la participación de las fuerzas vivas de los territorios en la creación institucional y en la formulación de políticas públicas en el marco del establecimiento de las regiones, y las leyes de descentralización de 1982 y 1983 consagraron esta participación institucionalizándola en los consejos económicos y sociales regionales, a los poderes públicos no les pareció bien actuar de la misma forma a nivel de las aglomeraciones urbanas. La participación del sector privado en la elaboración de las políticas de desarrollo urbano se ha dejado, en efecto, a la libre iniciativa de los actores locales y principalmente de los elegidos. Ciertamente, en algunas políticas sectoriales como las que se refieren a la planificación territorial (principalmente la elaboración de los nuevos Esquemas Directores), la ley requiere la consulta de las fuerzas económicas y sociales por medio de sus instancias representativas (principalmente las cámaras de comercio y los sindicatos de los trabajadores), pero ahí se acaban las obligaciones. En la práctica, a excepción de escasos ejemplos como los de Lille y Nantes presentados más arriba, los movimientos de las autoridades locales hacia las empresas no han ido más allá. Ha sucedido luego una carencia en el establecimiento de asociaciones necesarias para que las decisiones y las políticas de desarrollo urbano elaboradas por las entidades locales fueran, de una parte, legitimadas por las empresas y, de otra, pudieran ser puestas en marcha por los actores directamente implicados en esas políticas. Frente a esta carencia de los elegidos locales, las empresas y, principalmente, las más grandes, han preferido actuar por sí solas, haciendo operativas de este modo, así, unas opciones estratégicas que, a menudo, no eran las mismas que las de los poderes públicos (Jouve, 1999).

\section{¿Hacia una reforma global de las políticas de desarrollo urbano orquestada por el Estado?}

En 1998 se publicó un informe titulado «Demain la Ville». Este documento, más conocido como «informe Sueur» y por el nombre del presidente de la comisión que él dirige ${ }^{2}$, se elaboró a petición del Ministro de Asuntos Sociales con carácter previo a la creación de un nuevo ministerio de la ciudad. El informe Sueur es muy crítico con respecto a las políticas urbanas y especialmente con la política de la ciudad. Critica la zonificación de los programas condenando la así llamada discriminación positiva, denuncia la falta de coherencia de las políticas públicas y de los instrumentos de ordenación urbanística, así como la inestabilidad de las acciones del Estado y la escasez de medios financieros empleados en la lucha contra los problemas urbanos.

Este informe estableció «50 propuestas de actuación para el futuro de las ciudades». Subraya que la aglomeración urbana debe llegar a ser el lugar de las «decisiones de estructuración referentes a las ciudades». Por esta razón, pide que las áreas urbanas dispongan de ejecutivos elegidos por sufragio universal directo, que el Estado firme con esos ejecutivos contratos relativos a proyectos de estructuración y a las orientaciones de funcionamiento urbano, y que los distintos planes se articulen unos con otros. Exige la generalización de los comités de barrios para reforzar la democracia local. En el plano fiscal y financiero recomienda una extensión de la TPU para todas las aglomeraciones locales de más de 100.000 habitantes, la fungibilidad de los créditos de la política de la ciudad, su aumento y su permanencia votando una ley marco válida para diez años.

Aunque parece excesivo calificar este informe de innovador en la medida en que en resumidas cuentas no hace sino suscitar reflexiones y necesidades que se debaten desde hace varios años, tiene el mérito de tratar de hacerlas operativas e inmediatas. En cambio, es extremadamente conciso sobre el papel a atribuir a las empresas en las políticas de desarrollo urbano, contentándose con expresar el deseo de su implicación en las políticas públicas, a partir de su elaboración. 
Ciertamente el informe Sueur no va a ser puesto en marcha y el nuevo ministro de la ciudad, C. Bartolome, sólo recogerá algunas ideas. No obstante, este informe servirá de base para las reflexiones y discusiones en la elaboración de tres nuevas leyes sobre la ciudad y la ordenación del territorio que serán promulgadas en 1999 y 2000 y que, a este respecto, anuncian quizá un nuevo retorno del Estado y un deseo de retomar el problema del desarrollo urbano en su globalidad. En efecto, estas tres leyes contemplan una reforma completa de la acción del Estado y de las entidades locales sobre la ciudad: reforma institucional, reforma de la concepción de la acción pública y sus cuadros y reforma de los instrumentos de políticas públicas.

La ley sobre el fortalecimiento y la simplificación de la coo. peración intermunicipal, votada a finales de 1999 da paso a numerosas leyes sobre la cooperación intermunicipal y, especialmente, a la Ley ATR, la cual pone en marcha una reforma institucional de la cooperación intermunicipal, orientada, como su título indica, a simplificar la organización compleja de la intermunicipalidad francesa donde coexiste no menos de nueve tipos de EPCI con sus competencias, territorios y recursos diferentes. Esta Ley, llamada «Ley Chevènement» por el nombre del ministro del Interior de la época, crea tres nuevos EPCI (véase cuadro más abajo) que deben, dentro de un plazo, crear espacios de solidaridad a partir de la puesta en marcha de un proyecto común de desarrollo económico y de ordenación. Progresivamente estos tres EPCI reemplazarán a los existentes por transformación automática. A fin de permitir una mejor armonía económica y social de las aglomeraciones urbanas, la ley crea obligatoriamente un impuesto profesional único (TPU) en el territorio de las comunidades urbanas y de las comunidades de aglomeración urbana que representan, de hecho, los territorios urbanos, mientras que las comunidades de municipios se dirigen, principalmente, a las zonas rurales. Gracias a esta ley, las aglomeraciones urbanas francesas deberán progresiva pero rápidamente disponer de una organización política institucional simplificada, más clara para los habitantes, y de recursos financieros compartidos al nivel del territorio funcional de la ciudad. El cuadro que sigue representa de forma sintética el panorama institucional de la intermunicipalidad resultante de la ley.

Según el Ministerio del Interior (DGCL, 2001), los primeros resultados son más bien alentadores, con no menos de $90 \mathrm{comu}$ nidades de aglomeración urbanas creadas al 1 de enero de 2001 de las cuales 70 provienen de una transformación de otros EPCI. Además, el número de EPCI que tiene fiscalidad propia (principalmente la TPU) ha aumentado considerablemente pasando de alrededor de 1600 en 1998 a más de 2000 en la actualidad. Esta aparente vitalidad de las entidades locales no es, quizás, atribuible únicamente a una repentina toma de conciencia de la aglomeración urbana como territorio idóneo para las solidaridades y para las políticas de desarrollo por los elegidos locales. En efecto, el éxito de la ley podría igualmente explicarse por el importante señuelo financiero otorgado por el Estado, es decir, un aumento no despreciable de la Dotación Global de Funcionamiento (DGF), la principal subvención del Estado a las entidades locales, para los municipios que se reagrupen y adopten la TPU.

\begin{tabular}{|c|c|}
\hline $\begin{array}{ll} & \\
\text { Tipo de EPCI } & \text { Umbral } \\
\text { demográfico }\end{array}$ & $\begin{array}{l}\text { Fiscalidad } \\
\text { a nivel de la } \\
\text { aglomeración } \\
\text { urbana }\end{array}$ \\
\hline 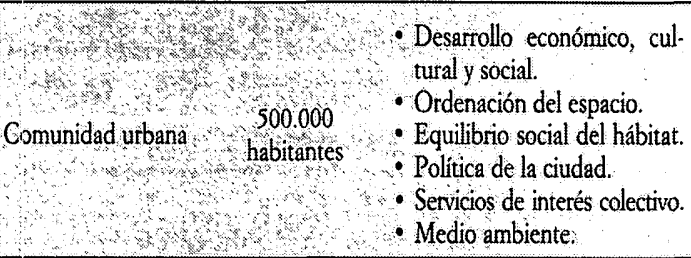 & $\begin{array}{l}\text { Obligatoria } \\
\text { IPU }\end{array}$ \\
\hline 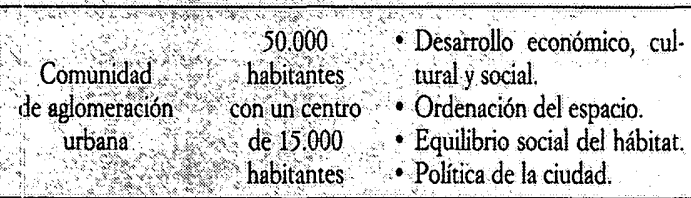 & $\begin{array}{l}\text { Obligatoria } \\
\text { TPU }\end{array}$ \\
\hline 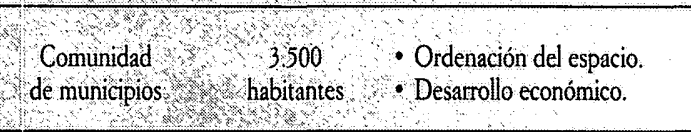 & $\begin{array}{l}\text { TPU posible } \\
\text { pero no } \\
\text { obligatoria }\end{array}$ \\
\hline
\end{tabular}

Más interesante para nuestro propósito es la nueva ley sobre la ordenación del territorio, porque presenta una reforma conceptual de las políticas públicas de desarrollo y de ordenación y crea un determinado número de instrumentos correspondientes. La reforma conceptual conlleva tres elementos: la identificación de un proyecto común con carácter previo a las ayudas del Estado, una contractualización de las políticas de aglomeración urbana en articulación con los otros territorios y la implicación de las fuerzas económicas y sociales para la elaboración y la puesta en marcha de los proyectos de desarrollo.

La Ley de Orientación para la Ordenación y el Desarrollo Permanente del Territorio (LOADDT) denominada Ley Voynet por el nombre del actual ministro de Medio Ambiente, está fuertemente marcada por la noción de «aglomeración urbana» que se expresa en tres términos: proyecto de aglomeración urbana, consejo de desarrollo y contrato de aglomeración urbana, que el Estado resume con una fórmula lapidaria: «un territorio, una estrategia, un proyecto, un contrato».

El territorio al que se dirige es el territorio considerado idóneo de las políticas públicas estratégicas. Se trata del territorio de las 140 áreas urbanas francesas identificadas en 1997 por el INSEE y que define los espacios funcionales urbanos (véase el texto siguiente). Estos territorios son igualmente los espacios de referencia de la Ley Chevènement, lo que hace que exista 

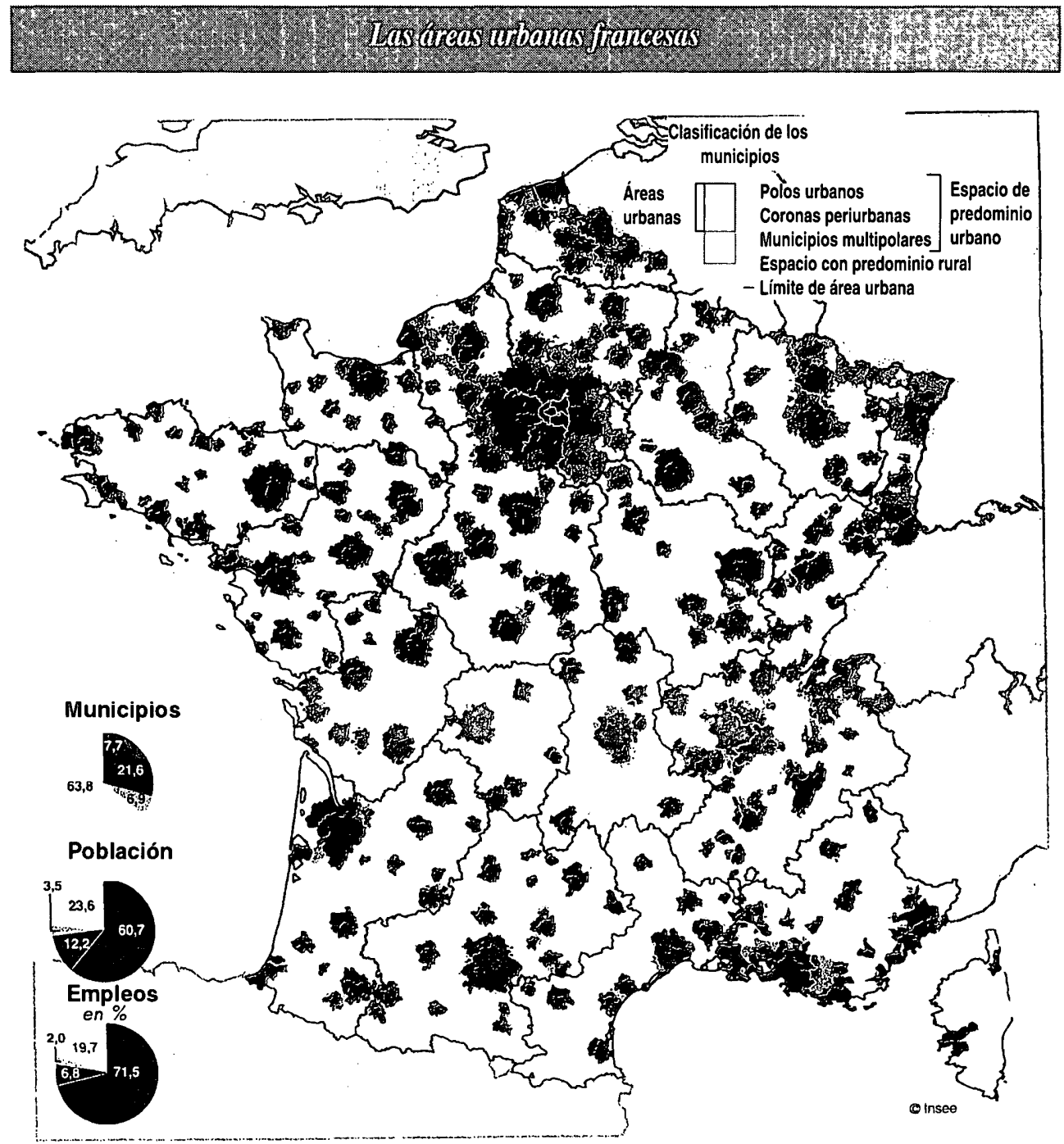

un vínculo directo entre la ley sobre la cooperación intermunicipal y la ley sobre la ordenación del territorio. La estrategia reenvía a los objetivos y a las prioridades determinadas por la aglomeración urbana, es decir, para la o las estructuras institucionales que la aplican. La formalización de esta estrategia se efectúa en el marco de un proyecto y es, principalmente, en este terreno donde la ley innova, vinculando la ayuda del Estado a la existencia de un proyecto de aglomeración urbana.

La LOADDT precisa, en efecto, que «el proyecto de aglomeración urbana es un proyecto de desarrollo fundado en una reflexión interdisciplinaria y que se basa en un diagnóstico compartido por el conjunto de fuerzas vivas del territorio en cuestión». Este proyecto debe «definir las orientaciones a medio y largo plazo fundadas en la identificación precisa de las políticas públicas a poner en marcha y de las iniciativas privadas a fomentar». Una vez que el proyecto es elaborado y aprobado, el Estado puede ayudar financieramente a la aglomeración urbana fir- mando con ella un contrato plurianual, llamado contrato de aglomeración. Para asegurar la coherencia, tanto en el contenido como en los medios financieros para llevar a cabo el proyecto, el contrato de aglomeración no será un documento aislado, sino que, por el contrario, estará integrado en el contrato de plan que el Estado firma cada seis años con la región (CPER). Por la misma razón, la región firma igualmente el contrato de aglomeración urbana. Con el establecimiento de un proyecto de aglomeración urbana y la firma de un contrato con el Estado, el área urbana se compromete - si es que no lo está ya- a dotarse en los años venideros de una estructura política, comunidad urbana o comunidad de aglomeración urbana según el tamaño de la mencionada aglomeración. Hay, pues, una complementariedad directa entre las dos leyes votadas en 1999.

El proyecto de aglomeración urbana se basa sobre un «diagnóstico compartido por las fuerzas vivas del territorio». A este respecto, la LOADDT innova igualmente estableciendo los con- 
sejos de desarrollo. Se trata de instancias obligatorias y consultivas que reúnen a los elegidos, medios socioprofesionales y asociativos en el territorio del área urbana. Estos consejos deberán ser consultados obligatoriamente para la elaboración, la aprobación y la puesta en marcha del proyecto de aglomeración urbana. La ley no impone ninguna organización de los consejos de desarrollo. Por el contrario, lo deja a la libre organización de los actores locales.

Todavía es demasiado pronto para hacerse una idea del alcance de la ley y, especialmente, de su impacto sobre la participación de los actores económicos en las políticas públicas de desarrollo. En efecto, aunque varios proyectos de aglomeración urbana han visto ya la luz en Lille, Lyon, Nantes o Burdeos, sólo se han firmado dos contratos de aglomeración urbana muy recientemente (las comunidades urbanas de Burdeos y de Creusot en diciembre de 2000). El primer consejo de desarrollo constituido en una gran ciudad ha visto la luz el 5 de febrero de 2001. Se trata del consejo del Grand Lyon. Reúne a 300 socios nombrados para un período de seis años. Los actores económicos ocupan un lugar importante, principalmente, en el seno del Comité Director, compuesto por 20 miembros, donde están representados la Cámara de Comercio y de Industria, el Movimiento de las Empresas de Francia (MEDEF) para las grandes empresas, la Confederación General de las Pequeñas y Medianas Empresas (CGGPME), la Joven Cámara Económica y el Club de Jóvenes Directivos. El consejo de desarrollo de Lyon está, pues, generosamente abierto, pero de su constitución no podemos sacar ninguna conclusión para las otras ciudades. Así pues, la noción de consejo de desarrollo puede tomar formas muy diferentes. Si bien la comunidad urbana de Lyon ha optado por una verdadera estructura de concertación, la metrópolis de Lille ha preferido elegir el procedimiento de debate, mucho menos formalizado, con ocasión de la aprobación de su esquema director en el 2000.

La reforma de los instrumentos de planificación y de las políticas de desarrollo urbano constituyen el tercer aspecto de la acción legislativa del Estado sobre el territorio urbano. Consciente de los desfases entre los documentos de urbanismo y de la obsolescencia de algunos de ellos, el gobierno de L. Jospin ha querido adaptar su contenido a la evolución de las ciudades y clarificar el sistema. En diciembre de 2000 ha sido promulgada la Ley «Solidaridad y Renovación Urbana» (Ley SRU) que pone fin al sistema de planificación instaurado por la LOF de 1967.

El objetivo primero de la ley, el más controvertido y que le da su nombre, es el de favorecer la mezcla social en el hábitat penalizando financieramente el rechazo de algunos municipios de acoger viviendas sociales. Pero más allá de este importante objetivo, la ley arregla el conjunto del sistema de planificación urbana. Así, ha suprimido los Esquemas Directores y los Planes de Ocupación del Suelo (POS) remplazándolos por los Esque- mas de Coherencia Territorial (SCOT) y los Planes Locales de Urbanismo (PLU).

Los SCOT sirven para definir la evolución de la aglomeración urbana y las prioridades en materia de vivienda, de comercio, de zonas de actividad y de transporte, mientras que los Esquemas Directores versaban esencialmente sobre el destino del suelo sin tener en cuenta las otras políticas a nivel de aglomeración urbana. Se trata, pues, de documentos (y de procedimientos de elaboración) que intentan articular las politicas que estaban anteriormente aisladas unas de otras por la vía de planes específicos (Planes de desplazamientos urbanos para los desplazamientos, Programas locales del hábitat para la vivienda, etc.). No habrá, pues, a nivel de aglomeración nada más que un documento único encargado de asegurar la coherencia territorial de las políticas públicas. Para ello, el SCOTT deberá basarse en «un objetivo de acondicionamiento y de desarrollo duradero» que deberá ser el proyecto de aglomeración urbana resultado de la Ley Voynet. Así pues, existe complementariedad entre estas dos leyes.

Los PLU suceden a los POS. Presentan el proyecto urbano del municipio en materia de ordenación, de tratamiento del espacio público, del paisaje y del medio ambiente. Más flexibles que los POS (permiten, en efecto, destinar varios usos a una misma zona), deben, como estos últimos, ser compatibles con los SCOT.

No hay obligación de requerir la participación de los actores económicos en la elaboración de los SCOT. Nada se dice en la Ley SRU sobre el papel de los consejos de desarrollo en la materia. La única obligación de información resulta del hecho que el SCOT debe ser sometido a información pública. Los actores económicos y sociales pueden intervenir únicamente en el marco de este procedimiento, pero una vez que el SCOT ha sido elaborado.

\section{Conclusión}

Las tres leyes votadas en 1999 y 2000 parecen completarse y ofrecer nuevos marcos y contenidos coherentes para la acción pública. Sin embargo, no se trata aquí de políticas fundacionales provenientes del Estado, es decir, que deberán ahora ser adoptadas por las entidades locales. El Estado aparece así como un Estado facilitador de la acción de las entidades locales, pero esto no parece suficiente para emprender una dinámica de cambio. Es preciso, igualmente, un Estado que dé ejemplo, es decir, que acepte reformarse, transformar su organización interna sobre la base de los nuevos territorios de acción pública que estas tres leyes intenta crear, es decir, el territorio de las áreas urbanas, en el sentido funcional del término (véase texto ante- 
rior). Ahora bien, una reforma tal no está asegurada. Está en marcha desde 1995 con el tema de los pays, pero por el momento ninguna dinámica real parece engranada, aun cuando el hecho de vincular contrato de aglomeración urbana y contrato de Estado-Región (CPER) representa un primer paso. Existe una duda real sobre la capacidad y la voluntad del Estado para autorreformarse. Por el momento, esto último queda a la expectativa, a mitad del camino entre el mantenimiento de un centralismo éclairé a la británica y una apertura hacia un federalismo a la italiana.

Existen dudas igualmente sobre la capacidad de las sociedades locales para abrir sus sistemas de actores, tanto a las empresas como al mundo de las asociaciones. Sobre este punto, los imperativos estimados de la competitividad internacional entre territorios no parecen llevar radicalmente a actores políticos y sociedad civil hacia colaboraciones estructurantes y estratégicas. Los consejos de desarrollo son una iniciativa acertada, pero permanecen tributarios de la cultura política francesa en la que lo público representa siempre el solo y único actor legítimo y, por ello, central en la elaboración y la conducción de las políticas de desarrollo local. A esta cultura política nacional vienen a unirse, frecuentemente, una historia local donde medios económicos y medios políticos no han hecho forzosamente buenas migas, y donde las veleidades recientes de los elegidos de lanzarse por sí solos a acciones de desarrollo económico han sido mal acogidas por los empresarios. El caso de Lyon es ilustrativo de esta situación, donde el consejo de desarrollo va a ser finalmente obligado a pronunciarse sobre un proyecto de aglomeración urbana ya muy elaborado por las autoridades públicas.

\section{Siglas utilizadas}

AMGVF: Asociación de los Alcaldes de las Grandes Ciudades de Francia

ATR: Administración Territorial de la República

CCI: Cámara de Comercio y de Industria

CDC: $\quad$ Caja de Depósitos y Consignaciones

CGPME: Confederación General de Pequeñas y Medianas Empresas
CPER: Contratos de Plan Estado Región

DATAR: Delegación para la Ordenación del Territorio y para la Acción Regional

DIV: Delegación Interministerial para la Ciudad

DSQ: $\quad$ Desarrollo Social de Barrios

DSU: Desarrollo Social Urbano

DTA: Directiva Territorial de Ordenación

EPCI: Establecimiento Público de Cooperación Intermunicipal

GPU: $\quad$ Gran Proyecto Urbano

INSEE: Instituto Nacional de Estadística y Estudios Económicos

LOADT: Ley de Orientación para la Ordenación y el Desarrollo del Territorio

LOADDT: Ley de Orientación para la Ordenación y el Desarrollo Permanente del Territorio

LOF: $\quad$ Ley de Orientación del Suelo

LOV: $\quad$ Ley de Orientación para la Ciudad

MEDEF: Movimiento de Empresas de Francia

PLH: $\quad$ Programas Locales del Hábitat

PLU: $\quad$ Planes Locales de Urbanismo

POS: $\quad$ Planes de Ocupación del Suelo

SCOT: Esquemas de Coherencia Territorial

SDAU: $\quad$ Esquemas Directores de Ordenación y de Urbanismo

SEM: $\quad$ Sociedad de Economía Mixta

SRU: $\quad$ Solidaridad y Renovación Urbana

TGV: $\quad$ Tren de Gran Velocidad

TPU: Impuesto Profesional Único

ZUP: $\quad$ Zonas de Urbanización Prioritaria 


\section{Bibliografía}

Association des Maires des Grandes Villes de France (AMGVF), Mon pays c'est la ville.

Commissariat Général du Plan (1993), «Décentralisation: l'âge de raison», Rapport du groupe de travail de préparation du XI plan, Documentation Française.

Commissariat Général du Plan (1993), Villes, Solidarité.

DATAR y AMGVF (2000), «Premiers enseignements de l'opération "Sites témoins" pour les contrats d'agglomération», rapport présenté aux Premières rencontres nationales des agglomérations, Clermont-Ferrand, marzo.

DGCL (Direction Générale des Collectivités Locales), consultation du site web: dgcl.gouv.fr, 12 de febrero de 2001.
Jouve, B. (1999), sobre Lyon.

JoUve, B., y LeFÈvre, C. (1999), Villes, Métropoles: les nouveaux territoires du politique, Paris: Anthropos.

LORRAIN, D. (1995), «La décentralisation ou l'action urbaine flexible», Sociologie du Travail.

LORRAIN, D., Les grands ensembliers.

MaBILeAu, A. (1991), Le système politique local, Paris: Montchrestien.

Sueur, J. P. (1998), Demain la ville, Paris: Documentation Française. 
